\title{
Nanocellulose: Beyond the Ordinary
}

\author{
Kojiro Uetani ${ }^{\mathrm{a}}$ and Takuya Kitaoka ${ }^{\mathrm{b}, *}$ \\ Nanocellulose is a collective term for nanoassemblies of macromolecular \\ cellulose in fibrous and crystalline forms, mainly originating from woody \\ bioresources. Fascinating physicochemical properties of nanocellulose, \\ such as high strength, light weight, transparency, and low thermal \\ expansion, have allowed development of nanocellulose-based functional \\ materials, but most of these materials face serious competition from \\ existing products. The inherent nanoarchitectures of nanocellulose cannot \\ be reconstructed by artificial means, and they are expected to contain \\ unknown functions that have not yet been achieved. Nanocellulose can \\ "run its own show" in the forthcoming sustainable society through \\ determining and highlighting its nanostructure-triggered novel material \\ functions that are beyond the ordinary.
}

Keywords: Nanocellulose; Nanocomposites; Thermal function; Light; Catalysis; Biomaterials

Contact information: a: The Institute of Scientific and Industrial Research (ISIR), Osaka University, 8-1 Mihogaoka, Ibaraki, Osaka 567-0047, Japan; b: Department of Agro-Environmental Sciences, Kyushu University, 744 Motooka, Nishi-ku, Fukuoka 819-0395, Japan;

* Corresponding author: tkitaoka@agr.kyushu-u.ac.jp

\section{Unlimited Potential of Nanocellulose}

Since the successful isolation of cellulose nanofibrils from wood pulp at the singlecomponent level in 2006 (Saito et al. 2006), research on nanocellulose, which can be defined as natural cellulosic nanomaterials that include both nanofibrillated cellulose (NFC) and cellulose nanocrystals (CNCs), has exploded. A number of nanocellulose-based composite materials have been developed because of the fascinating mechanical properties of nanocellulose, such as high strength, high Young's modulus, and low coefficient of thermal expansion, which originate from the extended chain crystal of nanocellulose. Lightweight and transparent nanocellulose films, which are called "nanopaper", have also been invented. However, most nanocellulose-based materials are faced with relentless competition from existing plastic-based materials, and there is a strong need to utilize the irreplaceable value of nanocellulose by exploiting the inherent nanostructures of nanocellulose that cannot be artificially reconstructed. In recent years, many challenges to break through the limits of current nanocellulose materials have been carried out for thermal conductive, optical, catalytic, and biomedical innovations. The clue must lie in discovering the unknown properties of nanocellulose to fulfill its unlimited potential.

\section{Thermal Control by Nanocellulose}

Cellulose is generally believed to be a typical thermal insulating material, and it is used as common utility goods, such as paper cups and corrugated boards, in our daily lives. The thermal insulation function of nanocellulose is manifested by partitioning air within the components. Nanocellulose forms porous aerogels with ultrafine voids, resulting in lower thermal conductivity than convective air. Nevertheless, it has been discovered that densely packed nanocellulose films exhibit 3 to 10 times higher thermal conductivity than 
plastic films (Uetani et al. 2015). Unlike the folded structures of general synthetic polymers, the extended chain crystals of nanocellulose have the potential to reduce the propagation loss of thermal vibrations, where the inherently strong and highly organized hydrogen bonds between nanocellulose chains significantly suppress the interfacial thermal resistance. Based on this new finding, various pioneering materials have been proposed using nanocellulose, including optically transparent and thermally conductive films, anisotropic heat transfer films, and thermal diffusivity modulation materials. High thermal conductivity nanocellulose is also promising as a matrix to improve the filler enhancement effect in a wide range of high thermal conductivity composites. Such biomass-based thermally conductive materials are expected to surpass the current high environmental impact products in future thermal management materials.

\section{Light Manipulation by Nanocellulose}

Since the development of transparent films composed of bacterial cellulose and acrylic resins, the optical applications of nanocellulose have been actively investigated. To further improve the transparency, surface smoothing of nonwoven nanocellulose films and birefringence matching with resins have been performed. Cellulose molecules exhibit high intrinsic birefringence, inspiring the design of optical manipulation materials with nanocellulose by nanofiber orientation technology. Rod-like nanocellulose, such as CNCs, self-assembles to form the Bouligand structure, which is often found in nature and exhibits cholesteric liquid crystal orientation. By combining self-assembly of nanocellulose with artificial manipulation, a number of optical materials have been reported to control the structural color and photonic properties. Application of three-dimensional (3D) printing to tune nanocellulose-aligned structures that cannot be achieved by only self-organization has shown promise in optoelectronics (see Fig. 1, Uetani et al. 2020). The artificially designed nanocellulose arrays are expected to enable advanced optical manipulation that is difficult to achieve with conventional plastic films.
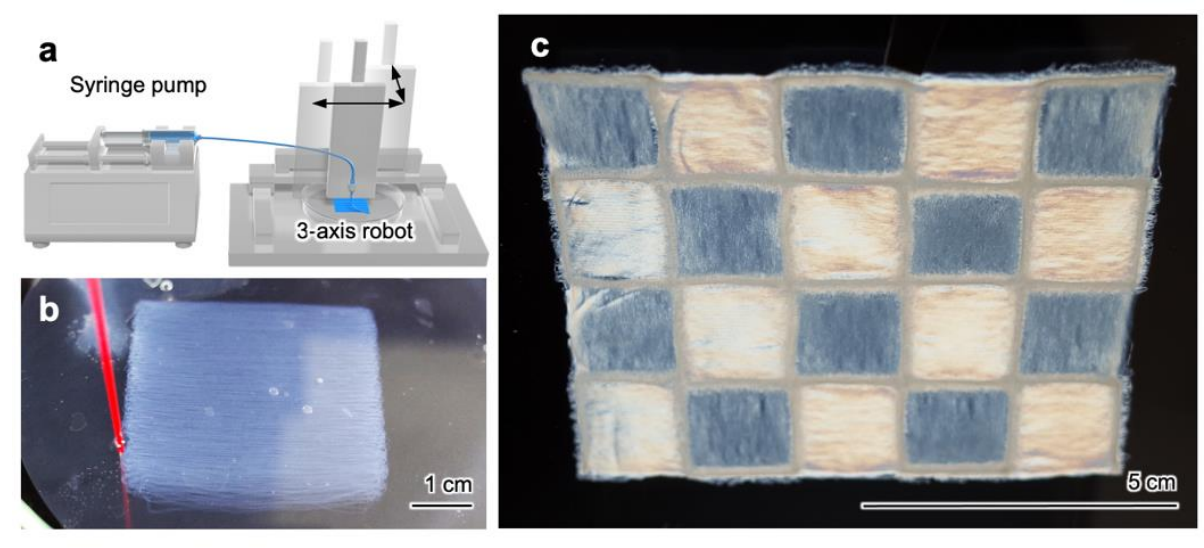

Fig. 1. Multi-axial oriented nanocellulose films prepared by liquid phase 3D patterning technology. (a) Oriented patterning mechanism of a nanocellulose suspension by a triaxial robot, (b) nanocellulose gel during patterning in acetone, and (c) checkered film with multi-axis oriented nanocellulose observed under crossed Nicols. Reproduced under the terms of the CC-BY 4.0 license (Uetani et al. 2020), copyright 2020, MDPI. 


\section{Interfacial Catalysis on Nanocellulose}

Highly efficient catalysis is a key technology to achieve green production of energy and materials, in which nanocellulose has been investigated as a catalyst carrier. In particular, metal (e.g., $\mathrm{Pt}, \mathrm{Pd}, \mathrm{Ru}, \mathrm{Ag}$, and $\mathrm{Au}$ ) nanoparticles deposited on the surfaces of nanocellulose have been successfully used in various catalytic reactions (Kaushik and Moores 2016); however, nanocellulose itself in metal-organic composites cannot play a starring role in catalysis. By contrast, several challenges in interfacial catalysis using nanocellulose have recently been reported, including chirality-specific hydrolysis of amino acids, highly efficient acetal hydrolysis, enantioselective hydrogenation of aromatic carbonyls, and enhanced proline-mediated organocatalysis with high enantioselectivity (Ranaivoarimanana et al. 2020). Nanocellulose has a regular alignment of chiral structures and an amphiphilic nature of its surface, and these will affect the fate of interfacial catalysis in stereochemistry, with expectations for the future. These trials are expected to provide new insight into nanocellulose-driven green catalysis, and they will expand the possibilities of nanocellulose for further advances in green sustainable chemistry and engineering.

\section{Biomedical Applications of Nanocellulose}

Cellulose and its derivatives have a long history of medical use, such as in dialysis for renal failure, wound dressings, and anti-bacterial composites. Likewise, nanocellulose has recently attracted increasing attention in biomedical applications (Jorfi and Foster 2015). Bacterial cellulose is a successful example because of its biocompatibility, hydrophilicity, gelling properties, and non-cytotoxicity. Conversely, nanocellulose from phytomass has not yet shown its potential. In current trials, the biomedical functions of nanocellulose composites with collagen, fibronectins, glycosaminoglycans, and other biocomponents in vivo only depend on the biological activities of its partners, not on those of nanocellulose. To determine new functions, we have to focus on the nanostructuretriggered singularity of nanocellulose. For example, 2,2,6,6-tetramethyl-piperidine 1-oxyl (TEMPO)-mediated oxidation of nanocellulose (Saito et al. 2006) can provide special nanofiber beyond the ordinary, which shows ordered nanostructures with regular alignment of carboxyl groups at the exposed C6 positions of the glucose units, highly crystalline long nanofibers of nanometer width, and an amphiphilic Janus form with surface asymmetry. Based on such unique characteristics, TEMPO-oxidized nanocellulose is a promising platform in current regenerative medicine to design cell culture scaffolds through determining the structures and functions of the extracellular matrix in vivo that play critical roles in cell attachment, proliferation, and differentiation.

\section{Future Opportunities}

Pioneering work on the extraordinary functions of nanocellulose will signal a new trend in forest nanoproduct industries, toward the Sustainable Development Goals (SDGs). From one of the alternatives to the one and only, we hope that nothing can take the place of nanocellulose.

\section{Acknowledgments}

The authors are grateful for support from the Grant-in-Aid for Scientific Research (KAKENHI) program (Grant Nos. JP19K22335 to K. U. and JP18K19233 to T. K.) of the Japan Society for the Promotion of Science. 


\section{References Cited}

Jorfi, M., and Foster, E. J. (2015). "Recent advances in nanocellulose for biomedical applications," J. Appl. Polym. 132(14), 41719. DOI: 10.1002/app.41719

Kaushik, M., and Moores, A. (2016). "Review: Nanocelluloses as versatile supports for metal nanoparticles and their applications in catalysis," Green Chem. 18(3), 622-637. DOI: $10.1039 / \mathrm{C} 5 \mathrm{GC} 02500 \mathrm{~A}$

Ranaivoarimanana, N. J., Habaki, X., Uto, T., Kanomata, K., Yui, T., and Kitaoka, T. (2020). "Nanocellulose enriches enantiomers in asymmetric aldol reactions," RSC Adv. 10(61), 37064-37071. DOI: 10.1039/d0ra07412h

Saito, T., Nishiyama, Y., Putaux, J.-L., Vignon, M., and Isogai, A. (2006). "Homogeneous suspensions of individualized microfibrils from TEMPO-catalyzed oxidation of native cellulose," Biomacromolecules 7(6), 1687-1691. DOI: $10.1021 / \mathrm{bm} 060154 \mathrm{~s}$

Uetani, K., Koga, H., and Nogi, M. (2020). "Checkered films of multiaxis oriented nanocelluloses by liquid-phase three-dimensional patterning," Nanomaterials 10 , 958. DOI:10.3390/nano10050958

Uetani, K., Okada, T., and Oyama, H. T. (2015). “Crystallite size effect on thermal conductive properties of nonwoven nanocellulose sheets," Biomacromolecules 16(7), 2220-2227. DOI: 10.1021/acs.biomac.5b00617 